\section{Расчет процессов заряда и разряда в тепловом накопителе энергии (Часть II)}

\author{
М. И. Куколев \\ Ю. К. Кукелев \\ Санкт-Петербургский государственный \\ политехнический университет \\ Петрозаводский государственный университет
}

\section{АННОТАЦИЯ}

Во второй части статьи (первая - в [2]) приводится решение уравнений теплообмена, описывающих процессы заряда и разряда теплового накопителя (ТН) с плавящимся теплоаккумулирующим веществом, размещаемым в ячейках основных конструктивных форм - пластины, цилиндра, сферы.

Ключевые слова: тепловые накопители, решение уравнений теплообмена.

\section{SUMMARY}

This paper presents a solving of the heat transfer equations at the charge-discharge processes in thermal storage systems with melting materials and different geometry of cells.

Keywords: heat transfer, melting, heat storage systems.

Частный случай - расчет процессов заряда и разряда ТН с ячейкой в форме пластины - был рассмотрен в [2]. В данной статье приводится более общий подход для ячеек основных конструктивных форм - пластины, цилиндра, сферы. Рассмотрим ТН последовательного включения (рис. 1) для предпускового подогрева ДВС [3].

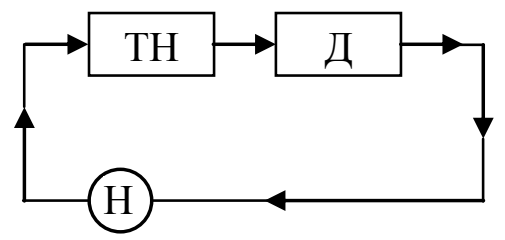

Рис. 1. Условная схема включения ТН:

ТН - тепловой накопитель, Д - двигатель;

$$
\text { Н - электронасос }
$$

Стрелками показано направление движения теплоносителя. При заряде нагретый в двигателе теплоноситель поступает в каналы ячеек ТН, где отдает теп-

\footnotetext{
${ }^{1}$ Авторы - соответственно доцент кафедры двигателей внутреннего сгорания СПбГПУ и доцент кафедры промышленной энергетики и энергосбережения ПетрГУ

() М. И. Куколев, Ю. К. Кукелев, 2003
}

лоту теплоаккумулирующему материалу (ТАМ). За счет плавления материала теплота накапливается. При разряде холодный теплоноситель поступает в каналы ячеек ТН, ТАМ затвердевает и отдает теплоту теплоносителю. Нагретый теплоноситель поступает за ТН на вход в холодный двигатель и нагревает его.

Перед записью систем уравнений, описывающих процессы заряда и разряда в ячейках тепловых накопителей (ТН) с плавящимся теплоаккумулирующим веществом, необходимо принять следующие допущения:

1) объем ТН постоянен;

2) изменения кинетической энергии и потенциальной незначительны;

3) параметры окружающей среды постоянны;

4) продолжительность процессов заряда и разряда конечна;

5) механизмами переноса теплоты в ТАМ являются теплопроводность и конвекция, доля переноса излучением незначительна и не учитывается;

6) процессы плавления и затвердевания ТАМ считаются одномерными;

7) перегрев и переохлаждение ТАМ отсутствуют, в исходном состоянии ТАМ имеет однородное распределение температуры $T_{m}^{*}$;

8) число Стефана для теплоаккумулирующего материала мало;

9) теплоподвод и теплоотвод осуществляются однофазным теплоносителем с постоянной теплопроводностью, плотностью и расходным теплосодержанием;

10) коэффициенты теплопередачи к ТАМ при заряде и от него при разряде постоянны;

11) процессы теплопередачи к ТАМ при заряде и от него при разряде считаются одномерными.

На рис. 2 и 3 приведены расчетные схемы процессов заряда и разряда ТН. Здесь обозначены: $T_{c i}^{*}$ - температура теплоносителя на входе в накопитель; $T_{c o}^{*}$ температура теплоносителя на выходе из накопителя; $T_{c w}^{*}$ - температура стенки емкости с теплоаккумулирующим материалом (принимается постоянной по всей нагреваемой поверхности $\left.F^{*}\left(x_{1}\right)\right) ; T_{m}^{*}, x_{1}^{*}$, $x_{2}^{*}$ - температура, начальная и конечная координаты границы фазового перехода “твердое тело - жидкость”. 


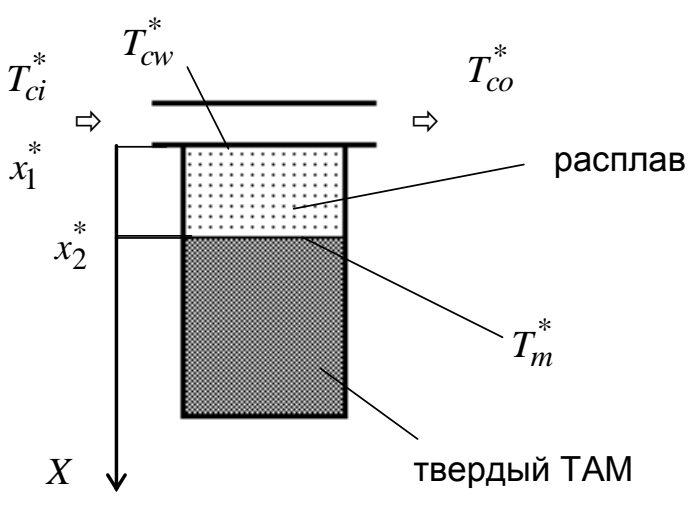

Рис. 2. Расчетная схема процесса заряда теплового накопителя последовательного включения с плавящимся теплоаккумулирующим материалом

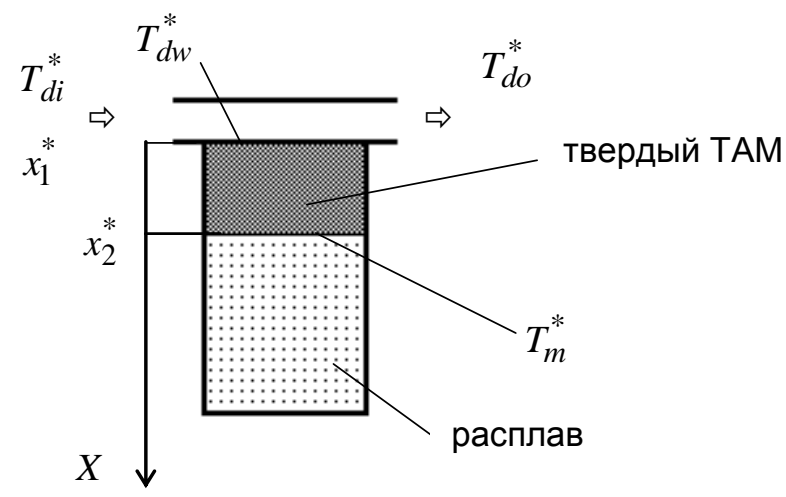

Рис. 3. Расчетная схема процесса разряда теплового накопителя

Для рассматриваемых схем справедливы следующие системы уравнений. Для заряда:

$$
\left\{\begin{array}{c}
Q_{c}^{*}=K_{c}^{*} F^{*}\left(x_{1}\right) \Delta T_{c}^{*} t_{c}^{*}, \\
K_{c}^{*} F^{*}\left(x_{1}\right) \Delta T_{c}^{*} t_{c}^{*} \eta_{c}=\frac{\lambda_{l}^{*}}{f^{*}\left(x_{1}, x_{2}\right)}\left(T_{c w}^{*}-T_{m}^{*}\right) t_{c}^{*}, \\
\frac{\lambda_{l}^{*}}{f^{*}\left(x_{1}, x_{2}\right)}\left(T_{c w}^{*}-T_{m}^{*}\right) t_{c}^{*}=\rho_{l}^{*} L^{*} F^{*}\left(x_{2}\right) w_{c}^{*} t_{c}^{*},
\end{array}\right.
$$

где $Q_{c}^{*}$ - подводимая энергия; $K_{c}^{*}$ - коэффициент теплопередачи; $F^{*}\left(x_{1}\right), F^{*}\left(x_{2}\right)$ - площади границы фазового перехода в начальный и конечный моменты времени; $\eta_{c}$ - энергетический КПД; $\Delta T_{c}^{*}$ - температурный перепад “теплоноситель - стенка”; $t_{c}^{*}$ - время заряда; $\lambda_{l}^{*}-$ коэффициент теплопроводности расплава; $\rho_{l}^{*}$ - плотность теплоаккумулирующего материала в расплавленном состоянии; $L^{*}$ скрытая удельная теплота фазового перехода; $w_{c}^{*}$ скорость передвижения границы фазового перехода.

Для разряда:

$$
\left\{\begin{array}{c}
\rho_{s}^{*} L^{*} F^{*}\left(x_{2}\right) w_{d}^{*} t_{d}^{*} \eta_{d}=\frac{\lambda_{s}^{*}}{f^{*}\left(x_{1}, x_{2}\right)}\left(T_{m}^{*}-T_{d w}^{*}\right) t_{d}^{*}, \\
\frac{\lambda_{s}^{*}}{f^{*}\left(x_{1}, x_{2}\right)}\left(T_{m}^{*}-T_{d w}^{*}\right) t_{d}^{*}=K_{d}^{*} F^{*}\left(x_{1}\right) \Delta T_{d}^{*} t_{d}^{*}, \\
K_{d}^{*} F^{*}\left(x_{1}\right) \Delta T_{d}^{*} t_{d}^{*}=Q_{d}^{*},
\end{array}\right.
$$

где обозначения аналогичны таковым в предыдущей системе, а индекс $d$ указывает на разряд, $s$ - на теплофизические характеристики теплоаккумулирующего материала в твердом состоянии.

Так как для накопления теплоты используется только плавление, то $q_{c}^{*}=\frac{d Q_{c}^{*}}{d t^{*}}=\frac{d H_{c}^{*}}{d t^{*}}=L^{*} \cdot \frac{d M_{l}^{*}}{d t^{*}}$. За время заряда происходит увеличение массы расплава $M_{l}^{*}=\rho_{l}^{*} \cdot w_{c}^{*} \cdot F^{*}\left(x_{1}\right) \cdot t_{c}^{*}$, следовательHO:

$$
q_{c}^{*}=L^{*} \cdot \rho_{l}^{*} \cdot \frac{d\left(w_{c}^{*} \cdot F^{*}\left(x_{2}\right) \cdot t_{c}^{*}\right)}{d t} .
$$

Продифференцировав, получаем:

$$
\begin{aligned}
& q_{c}^{*}=L^{*} \cdot \rho_{l}^{*} \cdot\left(F^{*}\left(x_{2}\right) \cdot t_{c}^{*} \cdot \frac{d w_{c}^{*}}{d t}+\ldots\right. \\
& \left.\ldots+w_{c}^{*} \cdot t_{c}^{*} \cdot \frac{d F^{*}\left(x_{2}\right)}{d t}+w_{c}^{*} \cdot F^{*}\left(x_{2}\right)\right) .
\end{aligned}
$$

Выделение энергии при разряде происходит за счет затвердевания жидкости: $q_{d}^{*}=L^{*} \cdot \frac{d M_{s}^{*}(t)}{d t}$. За время разряда происходит увеличение массы твердого вещества: $M_{s}^{*}(t)=\rho_{s}^{*} \cdot w_{d}^{*} \cdot F^{*}\left(x_{2}\right) \cdot t_{d}^{*}$, следовательно,

$$
q_{d}^{*}=L^{*} \cdot \rho_{s}^{*} \cdot \frac{d\left(w_{d}^{*} \cdot F^{*}\left(x_{2}\right) \cdot t_{d}^{*}\right)}{d t} .
$$


Продифференцировав, получаем:

$$
\begin{aligned}
& q_{d}^{*}=L^{*} \cdot \rho_{s}^{*} \cdot\left(F^{*}\left(x_{2}\right) \cdot t_{d}^{*} \cdot \frac{d w_{d}^{*}}{d t}+\ldots\right. \\
& \left.\ldots+w_{d}^{*} \cdot t_{d}^{*} \cdot \frac{d F^{*}\left(x_{2}\right)}{d t}+w_{d}^{*} \cdot F^{*}\left(x_{2}\right)\right) \cdot \eta_{d} .
\end{aligned}
$$

Согласно схеме включения ТН в систему охлаждения ДВС (рис. 1):

$$
\begin{gathered}
q_{c}^{*}=m_{c}^{*} \cdot c_{p}^{*} \cdot\left(T_{c i}^{*}-T_{c o}^{*}\right) \cdot \eta_{c}, \\
q_{d}^{*}=m_{d}^{*} \cdot c_{p}^{*} \cdot\left(T_{d o}^{*}-T_{d i}^{*}\right),
\end{gathered}
$$

и, с учетом введенных в первой части статьи безразмерных величин, системы уравнений преобразуются к безразмерному виду:

$$
\left\{\begin{array}{c}
\Theta_{c o}=1-\frac{N_{c} \cdot w_{c}}{\eta_{c}} \cdot R_{c}, \\
\Theta_{c o}-\Theta_{c w}=\frac{w_{c}}{\eta_{c}} \cdot R_{c}, \\
\Theta_{c w}=w_{c}^{2} \cdot \tau_{c} \cdot R_{c}
\end{array}\right.
$$

и

$$
\left\{\begin{array}{c}
\Theta_{d o}=1-N_{d} \cdot w_{d} \cdot \eta_{d} \cdot R_{d}, \\
\Theta_{d o}-\Theta_{d w}=w_{d} \cdot \eta_{d} \cdot R_{d}, \\
\Theta_{d w}=w_{d}^{2} \cdot \tau_{d} \cdot R_{d} .
\end{array}\right.
$$

Здесь $R_{c}, R_{d}$ обозначен комплекс:

$$
R=\frac{\Phi \cdot \tau \cdot \frac{d \Phi^{-1}}{d \tau}+1}{\Phi}
$$

для заряда и разряда.

Из систем уравнений находим безразмерную скорость передвижения границы фазового перехода при заряде и разряде:

$$
\begin{aligned}
& w_{c}=-0,5 \cdot \frac{N_{c}+1}{\tau_{c} \cdot \eta_{c}}+\left(\left(0,5 \cdot \frac{N_{c}+1}{\tau_{c} \cdot \eta_{c}}\right)^{2}+\ldots\right. \\
& \left.\ldots+\frac{1}{R_{c} \cdot \tau_{c}}\right)^{0,5}
\end{aligned}
$$

$w_{d}=-0,5 \cdot \frac{\left(N_{d}+1\right) \cdot \eta_{d}}{\tau_{d}}+\left(\left(0,5 \cdot \frac{\left(N_{d}+1\right) \cdot \eta_{d}}{\tau_{d}}\right)^{2}+\ldots\right.$

$\left.\ldots+\frac{1}{R_{d} \cdot \tau_{d}}\right)^{0,5}$

Формула справедлива для случая чистой теплопроводности. Если конструкция ячеек ТН позволяет развиться конвекции, что нежелательно с точки зрения снижения потерь энергии при хранении, то безразмерные скорости передвижения границы могут быть определены из следующих соображений [4].

Для теплового накопителя последовательного включения первые уравнения систем сохранятся:

$$
\begin{gathered}
\Theta_{c o}=1-\frac{N_{c} \cdot w_{c}}{\eta_{c}} \cdot R_{c}, \\
\Theta_{d o}=1-N_{d} \cdot w_{d} \cdot \eta_{d} \cdot R_{d} .
\end{gathered}
$$

Средний перепад температур в канале при заряде:

$$
\Delta T_{c}^{*}=\frac{T_{c i}^{*}-T_{c o}^{*}}{\ln \frac{T_{c i}^{*}-T_{c w}^{*}}{T_{c o}^{*}-T_{c w}^{*}}}
$$

и разряде:

$$
\Delta T_{d}^{*}=\frac{T_{d o}^{*}-T_{d i}^{*}}{\ln \frac{T_{d w}^{*}-T_{d o}^{*}}{T_{d w}^{*}-T_{d i}^{*}}}
$$

В случае конвекции температура стенки ячейки практически равна температуре плавления теплоаккумулирующего материала: $T_{c w}^{*} \approx T_{m}^{*}$ при заряде и соответственно $T_{d w}^{*} \approx T_{m}^{*}$ при разряде. Следовательно, $\Theta_{c w}=\Theta_{d w}=0$. Тогда

$$
\Delta T_{c}^{*}=\frac{T_{c i}^{*}-T_{c o}^{*}}{\ln \frac{1}{\Theta_{c o}}} \text { и } \Delta T_{d}^{*}=\frac{T_{d o}^{*}-T_{d i}^{*}}{\ln \Theta_{d o}}
$$

Как и в случае теплопроводности, будут справедливы соотношения при заряде:

$$
\begin{aligned}
& \frac{\Delta T_{c}^{*}}{T_{c i}^{*}-T_{m}^{*}}=\frac{w_{c}}{\Phi \cdot \eta_{c}} \cdot\left(\frac{\tau_{c}}{w_{c}} \cdot \frac{d w_{c}}{d \tau_{c}}+\ldots\right. \\
& \left.\ldots+\Phi \cdot \tau_{c} \cdot \frac{d \Phi^{-1}}{d \tau_{c}}+1\right)
\end{aligned}
$$


и разряде:

$$
\begin{aligned}
& \frac{\Delta T_{d}^{*}}{T_{m}^{*}-T_{d i}^{*}}=\frac{w_{d} \cdot \eta_{d}}{\Phi} \cdot\left(\frac{\tau_{d}}{w_{d}} \cdot \frac{d w_{d}}{d \tau}+\ldots\right. \\
& \left.\ldots+\Phi \cdot \tau_{d} \cdot \frac{d \Phi^{-1}}{d \tau}+1\right) .
\end{aligned}
$$

С учетом того, что

$$
\frac{\tau_{c}}{w_{c}} \cdot \frac{d w_{c}}{d \tau_{c}} \rightarrow 0 \text { и } \frac{\tau_{d}}{w_{d}} \cdot \frac{d w_{d}}{d \tau_{d}} \rightarrow 0,
$$

преобразовываем:

$$
\begin{gathered}
1-\Theta_{c o}=\frac{w_{c}}{\eta_{c}} \cdot R_{c} \cdot \ln \frac{1}{\Theta_{c o}}, \\
\Theta_{d o}-1=w_{d} \cdot \eta_{d} \cdot R_{d} \cdot \ln \Theta_{d o} .
\end{gathered}
$$

В то же время на основании первых уравнений систем можно получить:

$$
\begin{aligned}
& \Theta_{c o}=\exp \left(-N_{c}\right) \\
& \Theta_{d o}=\exp \left(-N_{d}\right) .
\end{aligned}
$$

Из равенств

$$
\begin{gathered}
1-\frac{N_{c} \cdot w_{c}}{\eta_{c}} \cdot R_{c}=\exp \left(-N_{c}\right), \\
1-N_{d} \cdot w_{d} \cdot \eta_{d} \cdot R_{d}=\exp \left(-N_{d}\right)
\end{gathered}
$$

получаем выражения для безразмерной скорости передвижения границы фазового перехода при заряде:

$$
w_{c}=\frac{1-\exp \left(-N_{c}\right)}{N_{c} \cdot R_{c}} \cdot \eta_{c}
$$

и разряде:

$$
w_{d}=\frac{1-\exp \left(-N_{d}\right)}{N_{d} \cdot R_{d} \cdot \eta_{d}} .
$$

Коэффициенты формы:

1. Для пластины $F^{*}\left(x_{1}\right)=F^{*}\left(x_{2}\right)$, следовательно: $\Phi_{\text {flat }}=1$;
2. Цилиндр и сфера при $\Phi=\frac{d_{1}^{*}}{d_{2}^{*}}$ и обозначении комплекса $\frac{K_{c}^{*} \cdot d_{c 1}^{*}}{2 \cdot \lambda_{l}^{*}}=m_{c}$ и $\frac{K_{d}^{*} \cdot d_{d 1}^{*}}{2 \cdot \lambda_{s}^{*}}=m_{d}$

имеют

$$
\Phi_{c y l_{c}}=e^{-\frac{w_{c, c y l} \cdot \tau_{c}}{m_{c}}} \text { и } \Phi_{s p_{c}}=1-\frac{w_{c, s p} \cdot \tau_{c}}{m_{c}}
$$

при заряде и

$$
\Phi_{c y l_{d}}=e^{-\frac{w_{d, c y l} \cdot \tau_{d}}{m_{d}}}, \Phi_{s p_{d}}=1-\frac{w_{d, s p} \cdot \tau_{d}}{m_{d}}
$$

при разряде.

Температуры стенки ячейки и на выходе из канала определяются по зависимостям для заряда:

$$
\begin{aligned}
& T_{c w}^{*}=T_{m}^{*}+\left(T_{c i}^{*}-T_{m}^{*}\right) \cdot w_{c}^{2} \cdot \tau_{c} \cdot R_{c}, \\
& T_{c o}^{*}=T_{c i}^{*}-\left(T_{c i}^{*}-T_{m}^{*}\right) \cdot \frac{N_{c} \cdot w_{c}}{\eta_{c}} \cdot R_{c}
\end{aligned}
$$

и разряда:

$$
\begin{gathered}
T_{d w}^{*}=T_{m}^{*}-\left(T_{m}^{*}-T_{d i}^{*}\right) \cdot w_{d}^{2} \cdot \tau_{d} \cdot R_{d}, \\
T_{d o}^{*}=T_{d i}^{*}+\left(T_{m}^{*}-T_{d i}^{*}\right) \cdot N_{d} \cdot w_{d} \cdot \eta_{d} \cdot R_{d} .
\end{gathered}
$$

При необходимости, если теплота ТН будет использоваться не только для предпускового разогрева ДВС, но и преобразовывается в полезную работу - к примеру, для создания пускового момента на валу при пуске двигателя или работы вспомогательных механизмов машины, - целесообразно оценить степень термодинамического совершенства проектируемого ТН с помощью эксергетического коэффициента полезного действия.

На этапе заряда он определяется как:

$$
\begin{aligned}
& \psi_{c, I}=\frac{T_{m}-1}{T_{m}} \cdot \eta_{c} \times \ldots \\
& \ldots \times \frac{T_{c i} \cdot A}{T_{c i} \cdot A+\eta_{c} \cdot \ln \left(1-A \cdot \eta_{c}^{-1}\right)},
\end{aligned}
$$

где $A=\left(1-\frac{T_{m}}{T_{c i}}\right) \cdot N_{c} \cdot w_{c} \cdot R_{c}$.

При разряде можно использовать следующую зависимость: 


$$
\psi_{d}=\frac{T_{m}}{T_{m}-1} \cdot R_{d} \cdot\left[\eta_{d}-\frac{\ln \left(1+A \cdot \eta_{d}\right)}{T_{d i} \cdot A}\right],
$$

где $A=\left(\frac{T_{m}}{T_{d i}}-1\right) \cdot N_{d} \cdot w_{d} \cdot R_{d}$.

В выражениях для $\psi_{c}$ и $\psi_{d}$ температуры представлены в безразмерном виде:

$$
T_{m}=\frac{T_{m}^{*}}{T_{d s}^{*}}, T_{c i}=\frac{T_{c i}^{*}}{T_{d s}^{*}}, T_{d i}=\frac{T_{d i}^{*}}{T_{d s}^{*}} .
$$

Таким образом, в соответствии с выбранной геометрией ячейки ТН с теплоаккумулирующим материалом рассчитывают $R, \tau$ и $w$. Затем определяют температуры стенок каналов $T_{c w}^{*}, T_{d w}^{*}$ и на выходе из ТН - $T_{c o}^{*}, T_{d o}^{*}$. По эксергетическим коэффициентам $\psi_{c}$ и $\psi_{d}$ оценивают степень термодинамического совершенства конструкции и принимают решение об использовании энергии ТН только в целях предпускового подогрева ДВС или еще и для преобразования в полезную работу.

Тому, как на практике использовать полученные соотношения для расчета предпускового подогрева ДВС по схеме, приводимой на рис.1, будет посвящена отдельная статья.

\section{СПИСОК ЛИТЕРАТУРЫ}

1. Куколев М. И., Кукелев Ю. К. Расчет процессов заряда и разряда в тепловом накопителе энергии (Часть I) // Труды лесоинженерного факультета ПетрГУ. Вып. 3. Петрозаводск: Изд-во ПетрГУ, 2001. С. 48 - 51.

2. Куколев М. И., Кукелев Ю. К., Луценко Л. А. Расчет термодинамической эффективности проектируемой ячейки теплового аккумулятора с помощью безразмерной скорости передвижения границы фазового перехода // Труды Второй российской национальной конференции по теплообмену. М.: Изд-во МЭИ, 1998. Т. 3. C. $206-209$.

3. Кукелев Ю. К., Куколев М. И. Тепловая подготовка лесовозных машин с помощью аккумуляторов тепла // Проблемы развития лесного комплекса Карелии: Тез. докл. республик. науч.-практ. конф. Петрозаводск: Изд-во ПетрГУ, 1996. С. $24-25$.

4. Куколев М. И. Основы проектирования тепловых накопителей энергии. Петрозаводск: Изд-во ПетрГУ, 2001. С. 83-86. 